\title{
Hereditary diffuse gastric cancer: genetics, prophylactic total gastrectomy, and follow up
}

This article was published in the following Dove Press journal:

Gastrointestinal Cancer:Targets and Therapy

I6 April 201 I

Number of times this article has been viewed

\author{
Santosh Shenoy' \\ Carl Palmer ${ }^{2}$ \\ Teresa Dunsworth ${ }^{3}$ \\ Stephen McNatt ${ }^{4}$ \\ 'Department of Surgery, Louis \\ A Johnson VA Medical Center, \\ Clarksburg, WV, USA; ${ }^{2}$ Department \\ of Medicine, West Virginia University, \\ General Internal Medicine and \\ Geriatrics, Morgantown, WV, USA; \\ ${ }^{3}$ General Internal Medicine and \\ Geriatrics, West Virginia University, \\ Morgantown, WV, USA; ${ }^{4}$ Department \\ of Surgery, Wake Forest University \\ Baptist Medical Center,Winston- \\ Salem, NC, USA
}

Background: Germline truncating mutations in the E-cadherin $(C D H 1)$ gene have been identified in families with hereditary diffuse gastric cancer (HDGC) and lobular breast cancers. This disease entity has an autosomal dominant pattern of inheritance with approximately $70 \%$ to $80 \%$ penetrance. Currently there is no definitive diagnostic modality for surveillance to detect this form of gastric cancer (GC) which carries a poor prognosis and high mortality. Prophylactic total gastrectomy is an option in the affected carriers and may offer improved long term survival for HDGC.

Methods: Two siblings from a single family with multi generation history of GC were identified as carriers of the mutation. After genetic counseling, they underwent laparoscopic prophylactic total gastrectomy with Roux-en-y esophagojejunostomy reconstruction. We describe the demographics and pedigree analysis, and postoperative and 5-year outcomes, and review the literature.

Results: Pathologic examination of the stomach revealed no foci of cancer in Patient 1; evidence of foci of gastric cancer was noted in Patient 2. Patient 2 subsequently developed lobular carcinoma of breast and underwent prophylactic bilateral mastectomy. No metastatic disease has been noted at 5-year follow up. She also had a successful pregnancy and birth of a healthy baby 3 years post gastrectomy. Both patients had a 30 to $40 \mathrm{lbs}$ ( 13.5 to $18 \mathrm{~kg}$ ) permanent weight loss. Both patients have maintained their preoperative activities and occupations.

Conclusion: HDGC is an uncommon hereditary form of GC with variable penetrance, $67 \%$ for men and $83 \%$ for women. Females were found to have a $39 \%$ risk for lobular breast cancer. Prophylactic total gastrectomy can be performed safely with low immediate and acceptable long term morbidity and low mortality. Serious consideration should be given to this procedure in asymptomatic carriers with $\mathrm{CDH} 1$ mutation. Female carriers of this mutation may be at increased risk for lobular carcinoma of the breast and should be monitored closely.

Keywords: gastric cancer, lobular breast carcinoma, $\mathrm{CDH1}$ gene

\section{Introduction}

Gastric cancer (GC) is the second most common cause of cancer deaths worldwide. ${ }^{1}$ There are two major types of gastric cancer: the intestinal type is sporadic and the more common variant. It is strongly associated with environmental risk factors, which include smoking, excessive alcohol consumption, dietary factors such as salted, smoked foods, and Helicobacter pylori infection. The other variant is diffuse gastric cancer (DGC) which occurs less commonly but has an infiltrative growth pattern with predisposition to early metastases and carries a worse prognosis.

The incidence of intestinal type has declined, but the incidence of DGC has remained stable or shows an increasing trend. The prognosis of patients with GC is
Correspondence: Santosh Shenoy

Department of Surgery, Louis

A Johnson VA Medical Center,

One Medical Center Dr., Clarksburg,

WV 26301, USA

Tel +I 3042762872

Fax +I 3046267754

Email shenoy2009@hotmail.com 
poor and carries a high mortality. The overall 5-year survival rate for all types and stages of gastric cancer in the United States from 1995 to 2000 was 23\% with a range of $58 \%$ in patients with localized disease to below $5 \%$ in patients with distant metastases. ${ }^{2}$

Hereditary diffuse gastric cancer (HDGC) is a familial form of gastric cancer due to germline mutations in the $\mathrm{CDH} 1$ gene located on chromosome 16. This familial syndrome has an autosomal dominant mode of inheritance and is caused by germline mutations in the E-cadherin (E-cad) gene $C D H 1$. This accounts for $1 \%$ of all cases of GC. ${ }^{3}$

This mutation was initially described by Jones in 1964 in a multigenerational Maori family from New Zealand. In 1998, Guilford et al identified the E-cad germline mutation in this family and other Maori families. ${ }^{3}$ Gayther et al subsequently reported similar $\mathrm{CDH} 1$ germline mutations in families of North European origin. ${ }^{4}$

We describe our experience and follow up with cases from a single family with HDGC.

\section{Methods}

Patient 1 on presentation was a 41-year-old male of North European ancestry with a family history of gastric cancer related deaths in multiple generations and was a confirmed $C D H 1$ mutation carrier. He was referred to us for prophylactic total gastrectomy. He was in good health without any previous medical or surgical history. The family history was significant for gastric cancer (Figure 1). The patient's mother was reported to have died of gastric cancer at age 52 years. The maternal grandfather was diagnosed and died of gastric cancer at 31 years. A maternal uncle is a confirmed

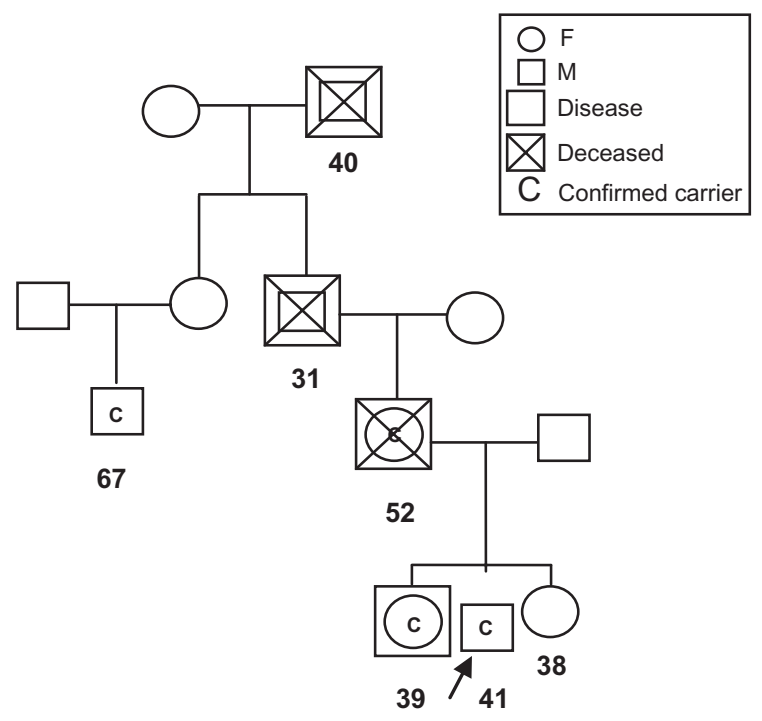

Figure I Family pedigree. Arrow points to patient I. carrier of the mutation and is 67 years old without evidence of gastric cancer.

The maternal great grandfather was diagnosed with gastric cancer and died of the disease at the age of 40 years. Given the extensive family history of gastric cancer, our patient's younger sibling sister elected to have genetic counseling and testing and was noted to have the frame shift mutation in exon 12, 1895 del AC of the $C D H 1$ gene.

This experience led to the genetic testing on our patient, who was confirmed to have a similar mutation. His other sibling sister is 38 years old and does not carry the mutation. Given the lack of specific screening for HDGC, prophylactic total gastrectomy was recommended. He underwent laparoscopic total gastrectomy with Roux-en-y esophagojejunostomy without intraoperative complications. The postoperative course was complicated with a minor superficial wound infection which was managed conservatively, and he was discharged home after 7 days in the hospital. He reports a $40 \mathrm{lbs}$ (18 kg) weight loss which stabilized at 4 years follow up and continues to follow a diet of small, frequent meals and is on vitamin B12 and iron supplements. He has resumed all his preoperative activities and continues his occupation as a fulltime academic physician. Final pathologic exam showed no evidence of carcinoma in the resected stomach.

Patient 2 is the female sibling of our first patient, who at the age of 39 years and after confirmation of the $C D H 1$ mutation underwent laparoscopic total gastrectomy with Roux-en-y esophagojejunostomy. Her postoperative course was uneventful and she was discharged after 10 days. She reports a $30 \mathrm{lb}(13.5 \mathrm{~kg})$ weight loss which has stabilized.

Her final pathology reported a small foci of T1 signet cell carcinoma in situ without lymph node involvement. Two years post gastrectomy she was diagnosed with lobular carcinoma of breast, confirmed on a breast mass noted on an MRI. She underwent bilateral prophylactic mastectomy and is free of any metastatic disease at 5 years follow up. She had a successful full term pregnancy and birth of a healthy baby 3 years following her gastrectomy. She also continues to have small, frequent meals with vitamin and iron supplements.

\section{Discussion Genetics}

The CDH1 gene is a tumor suppressor gene and maps to chromosome $16 \mathrm{q} 22.1$. This gene transcribes to encode for $120-\mathrm{kDa}$ protein called E-cadherin. This protein is a member of the transmembrane glycoprotein family expressed on epithelial tissues and is responsible for calcium dependent, 
cell to cell adhesion. Deregulation of this gene has been associated with enhanced initiation, infiltration, and metastatic ability of the tumor. This process occurs due to disruption of the cadherin-catenins complex with consequent loss of cell adhesion and result in increased cell motility. ${ }^{5-7}$

Approximately $40 \%$ of HDGC families may harbor this mutation. The penetrance for this mutation is reported to be in the range of $70 \%{ }^{8-10}$ Determining which $\mathrm{CDH} 1$ mutation carriers will develop DGC is not possible, unfortunately. Women carrying this mutation have an increased risk of lobular carcinoma of the breast. The cumulative risk of breast cancer approaches $39 \%$ by age 80 years, compared with the $12.5 \%$ lifetime risk for sporadic breast cancer. ${ }^{2,5,11-13}$

Carriers of the E-cad truncating germline mutations have a high lifetime risk of developing GC approaching a cumulative risk of $67 \%$ for men by the age of 80 years and $83 \%$ for women at comparable age. The average age of onset of gastric cancer is approximately 38 years and can range from 14 years to 82 years. In contrast sporadic gastric cancer occurs more commonly in men and in the fifth to sixth decade. ${ }^{9,11}$

The mutations noted and reported in the literature have been missense, nonsense, deletion, insertion, splicing and frameshift. ${ }^{14,15}$ These mutations have been observed in those of North American, North and Southern European, Japanese, Maori (New Zealand), Korean, Pakistani, Chinese, and Arabic ancestry. ${ }^{9,15}$ To date no $\mathrm{CDH} 1$ mutations has been identified in the patients with intestinal GC. ${ }^{4,13}$

Brooks-Wilson has published the revised criteria for consideration of $\mathrm{CDH} 1$ molecular genetic testing for $\mathrm{GC}$ based on a study of 42 families with HDGC: ${ }^{12}$

1. Family with 2 or more cases of GC with at least 1 patient with DGC diagnosed before age 50 years.

2. Three or more cases of gastric cancer in a family, diagnosed at any age, with at least 1 documented case of diffuse gastric cancer.

3. Family with more than 1 case of lobular carcinoma of the breast with or without DGC in first- or second-degree relatives.

4. Isolated case of DGC at age less than 35 years from low risk regions such as North America and Europe.

The New Zealand HDGC group recommends that family members should be offered genetic testing as early as age 16 years. Because genetic screening has medical, psychological, and economic consequences, counseling should be provided before genetic testing, and when reporting the results., 3

Genetic testing allows patients who carry the mutated CDH1 gene to make an informed decision on either surveillance endoscopy or prophylactic total gastrectomy to prevent gastric cancer. A blood sample from an affected member with HDGC is ideal, but because the disease is highly lethal no living members may be available for testing. In this situation the options are to offer screening to an obligate carrier, to other unaffected first degree family members, or to test DNA extracted from archival paraffin blocks of an affected family member. This option is often not possible because the DNA quality extracted may not be sufficient for screening of the whole gene. ${ }^{7}$

\section{Surveillance}

HDGC is characterized by the presence of multiple foci of diffuse type, signet ring cell carcinoma confined to the superficial gastric mucosa. Huntsman et al explained that most DGC in the resected stomachs after prophylactic gastrectomy are less than $1 \mathrm{~mm}$ in diameter and occur in apparently normal appearing gastric mucosa. Therefore these tumors are initially asymptomatic and are difficult to diagnose on routine endoscopy, causing delay in diagnosis with poor prognosis. ${ }^{16}$

Shaw et al described chromoendoscopy using congo redmethylene blue dye. This technique allows the detection of neoplastic foci of 4 to $10 \mathrm{~mm}$ in diameter but not foci less than $4 \mathrm{~mm}$. The authors concluded that chromoendoscopy with normal white light gastroscope facilitated detection of early gastric carcinoma foci not visible with routine endoscopy in patients with $\mathrm{CDH} 1$ mutations. ${ }^{17} \mathrm{~A}$ case report by van Kouwen et al demonstrated the use of [18f] fluoro-2-deoxy-D-glusose positron emission tomography scan (FDG-PET) in early detection of HDGC. In a single patient evaluated preoperatively, it was shown that 2 areas of increased signal intensity on PET scan, 1 in the fundus and 1 in the antrum correlated with foci of malignancy in the resected stomach. ${ }^{18}$

Endoscopic ultrasound is another tool and might be helpful in identifying early diffuse gastric cancer.

There are no official guidelines for the surveillance of E-cad germline mutation carriers; the general recommendations are upper endoscopy with random mucosal biopsies of fundus, body, incisura, and antrum of the stomach at 6- to 12-month intervals. ${ }^{7}$

\section{Prophylactic total gastrectomy}

Lewis and colleagues were among the first investigators to recommend prophylactic total gastrectomy for asymptomatic patients with $\mathrm{CDH} 1$ mutations. This recommendation was based upon their finding of occult DGC in the 
postgastrectomy specimens of 6 asymptomatic members of 2 families. $^{8}$

Huntsman et al described 5 individuals from 2 families with susceptibility to HDGC who underwent prophylactic total gastrectomy. In all 5 patients, neoplastic lesions that were undetected on gross examination were found only after pathological studies on resected stomachs. ${ }^{16}$

Norton et al reported occult DGC in $6 / 6$ patients with CDH1 mutation carriers who underwent prophylactic total gastrectomy with D2 lymph node dissection and Roux-en-y reconstruction. All resected stomach specimens had multiple foci of T1 invasive DGC with no lymph node involvement. ${ }^{19}$

Pharoah et al computed the risk for GC in $\mathrm{CDH} 1$ truncating mutation carriers to be $1 \%$ by the age of 20 years and up to $4 \%$ by the age of 30 years. ${ }^{11}$ Given this wide variation in the age of onset and the natural history of HDGC most authors recommend prophylactic gastrectomy for HDGC to be performed during the second decade of life in male mutation carriers, whereas female mutation carriers planning pregnancy will have to consider metabolic consequences associated with this procedure on pregnancy and may choose to delay the operation.

The prognosis for HDGC is poor, and most patients are diagnosed with advanced disease. Prophylactic total gastrectomy seems to be an appropriate option for the mutation carriers. There seems no role for chemotherapy or radiation therapy. ${ }^{3,9,16}$ After genetic counseling and confirmation of the mutation carrier status a complete preoperative medical work up should be done prior to prophylactic total gastrectomy. A baseline esophagogastroduodenal study should be performed with random biopsies of antrum, incisura, fundus, and the body of the stomach to identify foci of early GC.

Some authors also advocate a complete colonoscopy due to association of E-cad mutations with colon cancers.

A computed tomography (CT) scan of the abdomen and pelvis should be obtained to evaluate metastases.

Total gastrectomy ensuring removal of all gastric mucosa with Roux-en-y esophagojejunostomy with or without a pouch is the most widely accepted form of reconstruction. ${ }^{8,19,20}$ The procedure may be performed in a laparoscopic fashion by expert surgeons. Random controlled trials have shown that the incidence of anastomotic leak, stenosis, morbidity, and length of stay is not statistically different when a stapled vs a hand sewn anastomosis is performed. Lewis et al reported a leak rate of $12 \% .{ }^{8}$ A Japanese report showed a leak rate of $0.5 \%$ in patients with total gastrectomy for gastric cancer. ${ }^{21}$
The overall mortality of this procedure is reported to be as high as $22 \%$, but in patients with all forms of gastric cancer. Most of the sporadic forms of gastric cancer in the general population occur in older physiologically and nutritionally compromised patients which place them at a higher risk. Prophylactic total gastrectomy in HDGC is generally performed in younger patients who are otherwise healthy candidates.

Delay in diagnosis and untreated HDGC may have $100 \%$ mortality. Patients should be informed, however, that the accepted estimated mortality as a result of surgery is $0 \%-4 \%$. $^{7,8,22}$

Significant morbidity, however, is a common problem in patients undergoing total gastrectomy with reconstruction. There is a $10 \%$ to $15 \%$ permanent weight loss, due to decrease in eating capacity and increased transit due to loss of reservoir function. Patients should be followed by nutritional counselors in the pre- and postoperative periods and advised to consume small, frequent meals.

\section{Follow up}

Nearly all patients experience some extent of reflux, dumping, and diarrhea. ${ }^{14}$ These symptoms are most severe in the initial postoperative period and tend to improve with passage of time. Quality of life (QOL) studies indicate that patients have higher anxiety, depression, and loss of appetite with the initial diagnosis of gastric cancer, but return to normal gradually after surgery. Patients with total gastrectomy have worse intolerance to food and more weight loss compared with those undergoing subtotal gastrectomy. Younger, male patients have better QOL and outlook compared with female and older patients. Extensive lymph node dissections with D2 and D3 resections tend to have worse functional outcomes, but no significant effect on QOL. ${ }^{23,24}$

Other metabolic derangements noted after gastrectomy are malabsorption of nutrients including vitamin D, calcium, iron, and vitamin B12. Calcium is absorbed in the duodenum and due to reconstruction and change in anatomy leads to decreased absorption. Normal acid production is required for iron absorption. Loss of intraluminal acid leads to decreased iron absorption. Loss of intrinsic factor produced by the parietal cells in the stomach for vitamin B12 binding, leads to decreased absorption of this vitamin from the terminal ileum. Protein and fat absorption may be affected due to bacterial overgrowth and insufficient mixing of the food with the digestive enzymes leading to deficiency of fat soluble vitamins. All these patients will require lifelong vitamin supplementations. ${ }^{8,22,25,26}$ Female carriers should 
be advised of self breast examination, and to have annual mammograms and semiannual clinical breast examination beginning at least by age 30 as there is a high association of lobular breast cancer in $\mathrm{CDH} 1$ positive families. Some reports suggest routine bilateral breast MRI, as lobular breast cancer is known to elude mammographic detection. Prophylactic bilateral mastectomy may be considered as an option in CDH1 positive women to prevent breast cancers. ${ }^{5,13}$

\section{Conclusion}

HDGC belongs to the group of hereditary cancer syndromes and features increased risks of both gastric and breast cancers. The prognosis for HDGC is poor, and most patients are diagnosed with advanced disease. Prophylactic total gastrectomy seems to be an appropriate option for the mutation carriers and can be performed safely with low immediate and long term morbidity and mortality. Serious consideration should be given to this procedure in asymptomatic carriers with CDH1 mutation. Female carriers of this mutation may be at increased risk for lobular carcinoma of the breast and should be monitored closely.

\section{Disclosure}

The authors declare no conflicts of interest.

\section{References}

1. Alberts SR, Cervantes A, van de Velde CJH. Gastric cancer: epidemiology, pathology and treatment. Ann Oncol. 2003;14:ii31-ii36.

2. Jemal A, Siegel R, Ward E, et al. Cancer Statistics. CA Cancer J Clin. 2009;59:225-249.

3. Guilford P, Hopkins J, Harraway J, et al. E-cadherin germline mutations in familial gastric cancer. Nature. 1998;392:402-405.

4 Gayther SA, Gorringe KL, Ramus SJ, et al. Identification of germ-line E-cadherin mutations in gastric cancer families of European origin. Cancer Res.1998;58:4086-4089.

5. Schrader KA, Masciari S, Boyd N, et al. Hereditary diffuse gastric cancer: association with lobular breast cancer. Fam Cancer. 2008;7:73-82.

6. Suriano G, Oliveira C, Ferreira P, et al. Identification of CDH1 germline missense mutations associated with functional inactivation of the E-cadherin protein in young gastric cancer probands. Hum Mol Genet. 2003; $12: 575-582$.

7. Lynch HT, Silva E, Wirtzfeld D, et al. Hereditary diffuse gastric cancer: prophylactic surgical oncology implications. Surg Clin N Am. 2008;88: 759-778.

8. Lewis FR, Mellinger JD, Hayashi A, et al. Prophylactic total gastrectomy for familial gastric cancer. Surgery. 2001;130:612-617.
9. Guilford P, Blair V, More H, et al. A short guide to hereditary diffuse gastric cancer. Hered Cancer Clin Pract. 2007;5:183-194.

10. Lynch HT, Kaurah P, Wirtzfeld D, et al. Hereditary diffuse gastric cancer: diagnosis, genetic counseling, and prophylactic total gastrectomy. Cancer. 2008;112:2655-2663.

11. Pharoah $P$, Guilford $P$, Caldas $C$, et al. Incidence of gastric cancer and breast cancer in CDH1 (E-cadherin) mutation carriers from hereditary diffuse gastric cancer families. Gastroenterology. 2001;121: $1348-1353$.

12. Brooks-Wilson AR, Kaurah P, Suriano G, et al. Germline E-cadherin mutations in hereditary diffuse gastric cancer: assessment of 42 new families and review of genetic screening criteria. J Med Genet. 2004; 41:508-517.

13. Keller G, Vogelsang H, Becker, et al. Diffuse type gastric and lobular breast carcinoma in a familial gastric cancer patient with an E-cadherin germline mutation. Am J Pathol. 1999;55:337-342.

14. Caldas C, Carneiro F, Lynch HT, et al. Familial gastric cancer: overview and guidelines for management. J Med Genet. 1999;36:873-880.

15. Pedrazzani C, Corso G, Marrelli D, et al. E-cadherin and hereditary diffuse gastric cancer. Surgery. 2007;142:645-657.

16. Huntsman DG, Carneiro F, Lewis FR, et al. Early gastric cancer in young, asymptomatic carriers of germline E-cadherin mutations. NEJM. 2001;344:1904-1909.

17. Shaw D, Blair V, Framp A, et al. Chromoendoscopic surveillance in hereditary diffuse gastric cancer: an alternative to prophylactic gastrectomy? Gut. 2005;54:461-468.

18. Van Kouwen MCA, Drenth J, Oyen W, et al. [18F] Fluoro-2-deoxyD-glucose positron emission tomography detects gastric carcinoma in an early stage in an asymptomatic E-cadherin mutation carrier. Clin Cancer Res. 2004;10:6456-6459.

19. Norton JA, Ham CM, Van Dam J, et al. CDH1 truncating mutations in the E-cadherin gene: an indication for total gastrectomy to treat hereditary diffuse gastric cancer. Ann Surg. 2007;245:873-879.

20. Newman EA, Mulholland MW. Prophylactic gastrectomy for hereditary diffuse gastric cancer syndrome. J Am Coll Surg. 2006;202: 612-617.

21. Hyodo M, Hosoya Y, Hirashima Y, et al. Minimum leakage rate $(0.5 \%)$ of stapled esophagojejunostomy with sacrifice of a small part of the jejunum after total gastrectomy in 390 consecutive patients. Dig Surg. 2007;24:169-172.

22. Lehnert T, Buhl K. Techniques of reconstruction after total gastrectomy for cancer. Br J Surg. 2004;91:528-539.

23. Korenaga D, Orita H, Okuyama T, et al. Quality of life after gastrectomy in patients with carcinoma of the stomach. Br J Surg. 1992;79: 248-250.

24. Wu CW, Chiou JM, Ko F, et al. Quality of life after curative gastrectomy for gastric cancer in a randomized controlled trial. Br J Cancer. 2008; 98:54-59.

25. Buhl K, Lehnert T, Schlag P, et al. Reconstruction after gastrectomy and quality of life. World J Surg. 1995;19:558-564.

26. Hebbard PC, MacMillan A, Huntsman D, et al. Prophylactic total gastrectomy (PTG) for hereditary diffuse gastric cancer (HDGC): the Newfoundland experience with 23 patients. Ann Surg Oncol. 2009;16: 1890-1895.

Gastrointestinal Cancer: Targets and Therapy

\section{Publish your work in this journal}

Gastrointestinal Cancer: Targets and Therapy is an international, peer-reviewed, open access journal focusing on gastro-intestinal cancer research, identification of therapeutic targets and the optimal use of preventative and integrated treatment interventions to achieve improved outcomes, enhanced survival and quality of life for the

cancer patient. The manuscript management system is completely online and includes a very quick and fair peer-review system. Visit http://www.dovepress.com/testimonials.php to read real quotes from published authors. 